\title{
Thermodynamics of urban population flows
}

\author{
A. Hernando ${ }^{1}$ and A. Plastino $0^{2,3}$ \\ ${ }^{1}$ Laboratoire Collisions, Agrégats, Réactivité, Institut de Recherche sur les Systèmes Atomique et Moléculaires Complexes, \\ Université Paul Sabatier, 118 Route de Narbonne 31062, Toulouse CEDEX 09, France \\ ${ }^{2}$ National University La Plata, Physics Institute (IFLP-CCT-CONICET) C.C. 727, 1900 La Plata, Argentina \\ ${ }^{3}$ Universitat de les Illes Balears and IFISC-CSIC, 07122 Palma de Mallorca, Spain
}

(Received 21 June 2012; published 5 December 2012)

\begin{abstract}
Orderliness, reflected via mathematical laws, is encountered in different frameworks involving social groups. Here we show that a thermodynamics can be constructed that macroscopically describes urban population flows. Microscopic dynamic equations and simulations with random walkers underlie the macroscopic approach. Our results might be regarded, via suitable analogies, as a step towards building an explicit social thermodynamics.
\end{abstract}

DOI: 10.1103/PhysRevE.86.066105

PACS number(s): 89.65.-s, 05.70.-a, 89.75.Da

\section{INTRODUCTION}

\section{A. Universality classes in social systems}

The application of mathematical models to social sciences has a long and distinguished history [1]. One may speak of empirical data from scientific collaboration networks [2], citations of physics journals [3], internet traffic [4], Linux packages links [5], popularity of chess openings [6], as well as electoral results $[7,8]$, urban agglomerations $[9,10]$, and firm sizes all over the world [11]. An especially relevant issue is that of universality classes defined by to the so-called Zipf's law (ZL) in the cumulative distribution or rank-size distributions [3-6,9-15]. Maillart et al. [5] have found that links' distributions follow ZL as a consequence of stochastic proportional growth. Such growth assumes that an element of the system becomes enlarged proportionally to its size, being governed by a Wiener process. The class emerges from a condition of stationarity (dynamic equilibrium) [15]. ZL also applies for processes involving either self-similarity [6] or fractal hierarchy [13], all of them mere examples amongst very general stochastic ones [14]. A second universality class was found by Costa Filho et al. [7], who studied vote distributions in Brazil's electoral results. Therefrom emerge multiplicative processes in complex networks [8]. Such behavior ensues as well in (i) city-population rank distributions [16], (ii) Spanish electoral results [16], and (iii) the degree distribution of social networks [17]. As shown in Ref. [18], this universality class encompasses Benford's Law [19]. In the present vein, still another kind of idiosyncratic distribution is often reported: the log-normal one [20], which has been observed in biology (length and sizes of living tissue [21]), finance (in particular, the Black and Scholes model [22]), and firm sizes. The latter instance obeys Gibrat's rule of proportionate growth [23], which also applies to cities' sizes.

Together with geometric Brownian motion, there is a variety of models arising in different fields that yield Zipf's law and other power laws on a case-by-case basis [9,10,15,24-26], as preferential attachment [4] and competitive cluster growth $[8,17]$ in complex networks, used to explain many of the scale-free properties of social networks. For instance, we may mention detailed realistic approaches in urban modeling [27,28], opinion dynamics [29], and electoral results [7,30]. Of course, the renormalization group is intimately related to scale invariance and associated techniques have been fruitfully exploited in these matters (as a small sample see Refs. [26,31]).

It has been recently shown, in Ref. [32], that a variational principle based on the maximum entropy principle (MaxEnt) can be successfully applied to scale-invariant social systems. Used in the present context, it allows for a classification of the above-cited behaviors on the basis of inferences drawn from objective observables of the system. We had also shown in Ref. [33] that, including some dynamical information in the variational scheme [34], one is able to reproduce the shape of empirical city-population's distributions, going beyond the customary universality classes conventionally used in such regards. Indeed, a connection between explicit microscopic growth equations and the macroscopic characterization exists, illustrated for a logistic growth in Ref. [35]. We will here describe the manner in which the methods of that paper can be generalized to a first-principles theoretical framework describing population flows in terms of thermodynamic concepts.

\section{B. Motivation, statement of the problem, and goals}

We are looking here for more than models: what we aim for is to discover physical principles that may underlie some social phenomena. Our system is a specific geographical area whose population is distributed amongst several population nuclei (cities, villages, towns, etc.) Each nucleus's population is time dependent due to migration, birth, death, etc. Our aim is to quantitatively describe the population nuclei's variation. Microscopic variables are plentiful, but our main goal is to be able to identify macroscopic variables that can give a reasonable account of urban population variations.

We will proceed in seven steps, as indicated in the scheme below.

(i) Introduce the basic observables and the empirical data sets.

(ii) Identify the stochastic nature of the city-population growth rates.

(iii) Postulate dynamic microscopic equations and empirically validate them.

(iv) Perform numerical experiments with random walkers following these dynamical equations. Parameterize the macroscopic diffusion process for free evolution or evolution under constraints. 
(v) Show that equilibrium configurations of such evolution can be predicted by the maximum entropy principle, using few macroscopic parameters.

(vi) Derive thermodynamiclike relations between these macroparameters.

(vii) Exhibit the applicability of our thermodynamic description by modeling empirical urban flows as a scale-invariant ideal gas.

The paper is organized as follows. Step (i) is addressed in Sec. II. Section III deals with step (ii), Sec. IV with step (iii), Sec. V with step (iv), and Sec. VI with steps (v) and (vi). Finally, applications are dealt with in Sec. VII. Discussions are given in Sec. VIII, and we conclude in Sec. IX.

\section{PRELIMINARY MATTERS}

The basic ingredients we need in our approach, following Refs. [33,34], are

(a) $n$, the total number of "population-nuclei";

(b) $x_{i}(t)$, the population of the $i$ th nucleus at time $t$ (and $\mathbf{x}(t)=\left\{x_{i}(t)\right\}_{i=1}^{n}$ a vector with all the populations). Since we are interested in populations of the order of hundreds and millions, we will consider, following the example of the references cited above, the continuous limit of this variable, with no significant loss of accuracy.

(c) $x_{0}$ and $x_{M}$, the minimum and maximum allowed nucleus's population (in general $x_{0}=1$ and $x_{M}=\infty$ );

(d) $N_{T}$, the total area's population $\left[N_{T}=\sum_{i=1}^{n} x_{i}(t)\right]$;

(e) $\dot{x}_{i}(t)$, the time derivative of $x_{i}(t)$ [thus the pairs $\left\{\left(x_{i}, \dot{x}_{i}\right)\right\}_{i=1}^{n}$ compose the "urban phase space"]; and

(f) some a priori knowledge of the dynamics at hand, expressed via

$$
\dot{x}_{i}(t)=k_{i}(t) g_{i}[\mathbf{x}(t)]
$$

where $g_{i}$ are population-functions to be determined and $k_{i}(t)$ growth rates independent of the $g_{i}$. This hypothesis about the dynamics will be empirically justified below.

The raw data used in our analysis are mainly obtained from the Spanish state institute INE [36] and cover annually the period 1996-2010 (with the exception of 1997). It encompasses 8116 municipalities (the smallest Spanish administrative unit) distributed within 50 provinces (the building blocks of the autonomous communities). We use provinces and municipalities as the closest representatives of the ideal of a closed system's fundamental elements. Other regions of the world are also used as examples. In this scenario, the total population $N_{T}$ of a province is distributed into $n$ nuclei. The $i$ th nucleus accounts for a population of $x_{0} \leqslant x_{i}(t) \leqslant x_{M}$ at time $t$. The pertinent time evolution obeys Eq. (1).

\section{STOCHASTIC NATURE OF POPULATION GROWTH RATES}

We begin dealing with step (ii) of our scheme. We focus attention on the form of the growth rates $k_{i}(t)$ of Eq. (1). The value of $k_{i}$ above depends upon millions of individual decisions so that one would expect stochastic, a priori unpredictable, behavior. However, we will show in this section that: given the form of Eq. (1), the growth rates $k_{i}$ are population-independent; and some level of universality underlies these rates.
We start by considering an arbitrarily small time window $\delta t$ around $t$ (of the order of a few years). We define the time average of the population change for the $i$ th city as $m_{i}=\left\langle\dot{x}_{i}(t)\right\rangle_{\delta t}=\left\langle k_{i}(t) g_{i}(t)\right\rangle_{\delta t}$, and its variance as $s_{i}^{2}=$ $\left\langle\left(k_{i}(t) g_{i}(t)-m_{i}\right)^{2}\right\rangle_{\delta t}$. We assume now that the variation of the functions $g_{i}$ in the time window for which one evaluates the pair $m_{i}-s_{i}$ is negligible with respect to the variation of the growth rates (a fact that will be justified a posteriori below, after defining the form of the $g_{i}$ functions). This is equivalent to assuming that

$$
\sigma_{k_{i}}^{2}\left\langle g_{i}(t)\right\rangle_{\delta t}^{2} \gg\left\langle k_{i}(t)\right\rangle_{\delta t}^{2} \sigma_{g_{i}}^{2},
$$

where $\sigma_{k_{i}}^{2}$ and $\sigma_{g_{i}}^{2}$ are the variances of $k_{i}$ and $g_{i}$, respectively. We write then

$$
\begin{gathered}
m_{i} \approx\left\langle k_{i}(t)\right\rangle_{\delta t} \times\left\langle g_{i}(t)\right\rangle_{\delta t}, \\
s_{i}^{2} \approx \sigma_{k_{i}}^{2}\left\langle g_{i}(t)\right\rangle_{\delta t}^{2} .
\end{gathered}
$$

We study now the distribution of a new variable defined via the scaling law $\xi_{i}(t)=\left(\dot{x}_{i}(t)-m_{i}\right) / s_{i}$, immediately finding

$$
\xi_{i}(t)=\frac{k_{i}(t)-\left\langle k_{i}(t)\right\rangle_{\delta t}}{\sigma_{k_{i}}} .
$$

The variable $\xi_{i}(t)$ has null average and unit standard deviation, but the shape of the distribution $p_{\Xi}(\xi)$ must be the same of that of the growth rate $k$. If our assumptions are correct, the shape of these distributions should not depend upon $x_{i}(t)$. We have verified the hypothesis, as our first result here, with reference to all of Spain's municipalities in the time window $\delta t=15$ years. Indeed, we have calculated the $\xi$ quantiles of the $p_{\Xi}$ distribution as a function of the population (top panel Fig. 1). No apparent $x$ dependence can be detected. The shape of the overall distribution $p_{\Xi}(\xi)$ looks like a normal one

$$
p_{\Xi}(\xi)=\frac{e^{-\xi^{2} / 2}}{\sqrt{2 \pi}},
$$

with cumulative distributions of the form

$$
P_{\Xi}(\xi)=\frac{1}{2}\left[1+\operatorname{erf}\left(\frac{\xi}{2}\right)\right] .
$$

Save for some fluctuations, we have not found, for the different provinces (bottom panel Fig. 1), any dependence on the shape of $p_{\Xi}(\xi)$. Changing the time window $\delta t$ and the reference time $t$ does not change these results. Further, note that they are seemingly independent of the population, the geographical area, or the time, so that one might speak, with a grain of salt, of "universality" (our second result here).

Consequently, we will consider herefrom that our variable $\xi$ can be regarded as that of a stochastic process simulated via the standard Brownian motion. For the sake of simplicity, we assume in the following that the growth rates do not exhibit significant correlations and that can be reproduced by a Wiener process (we discuss in detail this issue in Sec. VIII).

\section{INTRODUCING MICROSCOPIC EQUATIONS OF MOTION}

\section{A. Proportional growth}

We are now at step (iii). We will, for the sake of simplicity, assume (and a posteriori justify), that $g_{i}$ 's shape depends only 

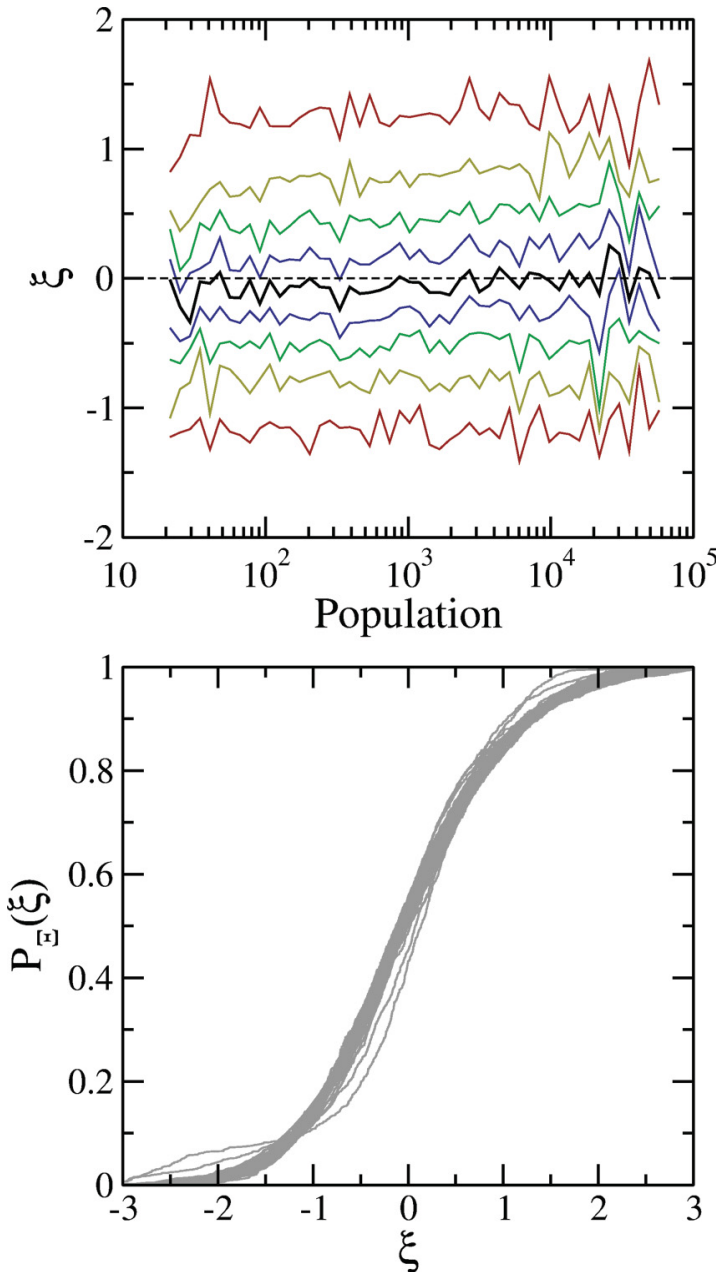

FIG. 1. (Color online) Top: quantiles from 0.1 (red) to 0.9 (blue) (each of 0.1 ) for the distribution of the scaled growth rates $p_{\Xi}(\xi)$ (see text) as a function of the population $x$ (the median is shown in black). Bottom: cumulative distribution of the scaled growth rates $P_{\Xi}(\xi)$ for each of Spain's provinces.

on its own $x_{i}$ 's population, (i.e., $g_{i}[\mathbf{x}(t)] \simeq g_{i}\left[x_{i}(t)\right]$ ). In order to obtain an educated guess for the explicit analytical form, we appeal to a successful cluster-growth model in networks $[8,17]$, before describing city-population distributions. We consider first a network of nodes (that eventually represents the social network) and a single node as seed of a cluster. Initially, the first neighbors of the seed will belong to the cluster with a given probability $P(t=0)$. At a subsequent time $t$, the first neighbors of the members of the cluster become also members with probability $P(t)$. Proceeding in this vein, it is reasonable to conjecture that the time variation of the cluster size $\dot{x}$ at time $t$ acquires the form

$$
\dot{x}(t)=\sum_{j=1}^{x(t)} P(t) c_{j}(t),
$$

where $c_{j}(t)$ is the number of first neighbors associated to node $j$ at time $t$. We appeal now to the central limit theorem to write

$$
\dot{x}(t)=P(t)\left[\bar{c}(t) x(t)+\sigma_{c}(t) \sqrt{x(t)} \chi(t)\right] .
$$

Here $\bar{c}(t)$ is the mean neighbor's number at time $t, \sigma_{c}(t)$ its standard deviation, and $\chi(t)$ an independent normally distributed number. This last summand, usually neglected for very large sizes, is associated to finite-size effects. The first term, size proportional, generates proportional growth. In view of this result, we consider the form

$$
g_{i}\left(x_{i}\right)=\left[x_{i}\right]^{\alpha}
$$

with $\alpha=1$ or $1 / 2$. Considering then both terms in the microscopic dynamics we write

$$
\dot{x}_{i}(t)=k_{1 i}(t) x_{i}(t)+k_{\frac{1}{2} i}(t) \sqrt{x_{i}(t)},
$$

where $k_{1 i}(t)$ and $k_{\frac{1}{2} i}(t)$ are two (a priori) independent Wiener coefficients. This dependence is checked out by comparison of the previously employed $s_{i}$ numbers with a functional form of the type

$$
\begin{aligned}
s_{i}^{2}\left(x_{i}\right) & =\left\langle\left[\dot{x}_{i}\right]^{2}-\left\langle\dot{x}_{i}\right\rangle_{\delta t}^{2}\right\rangle_{\delta t} \\
& =\sigma_{1 i}^{2} x_{i}^{2}+\sigma_{\frac{1}{2} i}^{2} x_{i},
\end{aligned}
$$

where $\sigma_{1 i}$ and $\sigma_{\frac{1}{2} i}$ are the associated deviations of $k_{1 i}$ and $k_{\frac{1}{2} i}$, respectively. Rewriting (12) in a more convenient way we have

$$
s_{i}^{2}\left(x_{i}\right) / x_{i}=\sigma_{1 i}^{2} x_{i}+\sigma_{\frac{1}{2} i}^{2},
$$

which, for sizes small enough reduces to

$$
s_{i}^{2}\left(x_{i}\right) / x_{i} \approx \sigma_{\frac{1}{2} i}^{2},
$$

while for very large sizes one has

$$
s_{i}^{2}\left(x_{i}\right) / x_{i} \approx \sigma_{1 i}^{2} x_{i} .
$$

The transition between these two regimes should take place at a value $x_{T}=\sigma_{\frac{1}{2} i}^{2} / \sigma_{1 i}^{2}$. Figure 2 displays, as our

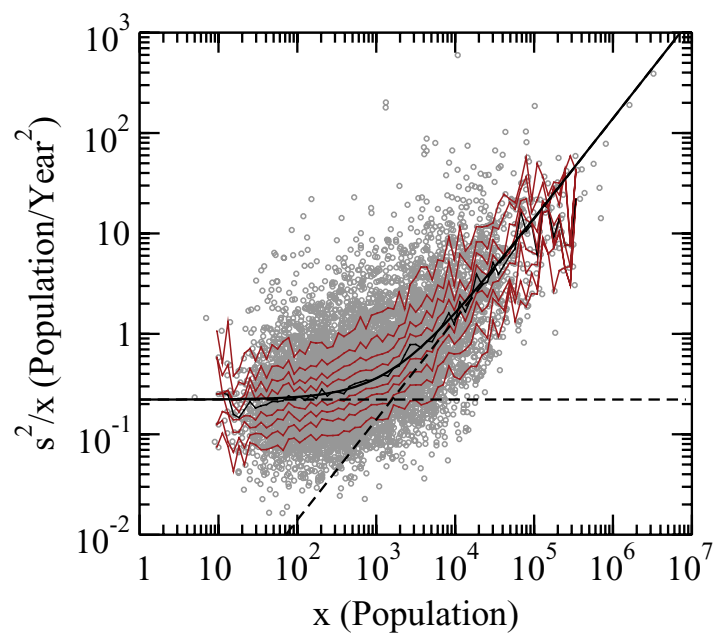

FIG. 2. (Color online) Variance $s^{2} / x$ vs population $x$ (see text), each dot representing one municipality. Red lines: quantiles from 0.1 to 0.9 (each of 0.1 ). Solid black line: fit to the median value following Eq. (13). Dashed black lines: extrapolation of the finite-size term [Eq. (14)], and extrapolation of the multiplicative term [Eq. (15)]. 
third result, the $\left[x_{i}, s_{i}^{2}(x) / x_{i}\right]$ pairs for all the Spanish municipalities, together with appropriate quantiles. The median $\operatorname{med}\left[s_{i}\left(x_{i}\right) / x_{i}\right]$ nicely fits things with $\sigma_{1 i}=0.0119$ years $^{-2}$ and $\sigma_{\frac{1}{2} i}=0.47$ population years ${ }^{-2}$. We appreciate the fact that finite-size fluctuations are larger than multiplicative ones, the later dominating, of course, for large sizes. Our transition occurs at population values of the order of 1500 inhabitants. Surprisingly enough, the distribution of the variable $s_{i}^{\prime}=$ $\ln \left[s_{i}\left(x_{i}\right) / \sqrt{x_{i}}\right]-\ln \left[\operatorname{med}\left(s_{i}\left(x_{i}\right) / \sqrt{x_{i}}\right)\right]$ becomes independent of $x_{i}$, being of a Gaussian nature.

At this point, we need still to address two further questions. The first of them concerns the validity of the assumption (16), made to derive Eq. (5). For cities with a population $>1500$ inhabitants, we use $g_{i}(t)=x_{i}(t)$, so that the condition can be recast as

$$
\frac{\left\langle\left(\dot{x}_{i} / x_{i}\right)^{2}\right\rangle_{\delta t}}{\left\langle\dot{x}_{i} / x_{i}\right\rangle_{\delta t}^{2}} \frac{\left\langle x_{i}\right\rangle_{\delta t}^{2}}{\left\langle x_{i}^{2}\right\rangle_{\delta t}} \gg 1 .
$$

Using the empirical data for a time window of 15 years, we have found that the mean value of the left-hand side is $6 \times 10^{15}$ (with $9 \times 10^{11}$ for the geometric mean), a result that justifies our assumption. We have also verified it, for cities containing less than 1500 inhabitants, by using $g_{i}(t)=\sqrt{x_{i}(t)}$ instead, with analogous results.

Our second issue revolves around the time averages of the growth rates. The finite-size term's average is $\left\langle k_{\frac{1}{2} i}(t)\right\rangle_{\delta t}=0$ by definition, but this is not so for the multiplicative term $\left\langle k_{1 i}(t)\right\rangle_{\delta t} \neq 0$, that is a priori regarded as constant and size independent. This is indeed empirically true on occasion, but not always. For instance, such assumption cannot account for the migration from the countryside to big cities. To reproduce such behavior, the mean relative growth should depend on the population. We intend to tackle this issue below.

\section{B. Taking into account internal flow}

It is a fact that small populations tend to diminish while large towns tend to increase their population. We encounter this scenario for most of the 50 provinces of Spain, as discussed in Ref. [33]. We will appeal below to the line of reasoning presented there, that we repeat here for the sake of completeness.

This effect can be described by recourse to a smooth dependence of the mean relative growth $\left\langle\dot{x}_{i} / x_{i}\right\rangle$ on $\ln (\langle x\rangle)$ that generates what we will call internal flow. A second-order expansion in $\ln (\langle x\rangle)$ reads

$$
\langle\dot{x} / x\rangle \simeq a+b \ln (\langle x\rangle)+c \ln (\langle x\rangle)^{2}
$$

where the values of $a, b$, and $c$ are derived from the corresponding Taylor coefficients. Assuming $b \gg c$ we can safely write

$$
\langle\dot{x} / x\rangle \simeq\left\langle k_{1}\right\rangle+\left\langle k_{q}\right\rangle[\langle x\rangle]^{q-1},
$$

where we have defined, for convenience sake, $\left\langle k_{1}\right\rangle=a-$ $b^{2} / 2 c,\left\langle k_{q}\right\rangle=b^{2} / 2 c$, and $q-1=2 c / b$. To validate our assumptions, we fitted empirical provincial data to Eq. (18) via $\left\langle k_{1}\right\rangle,\left\langle k_{q}\right\rangle$, and $q$. This was done whenever it was feasible, since, in some cases, a quasilinear relation is found that generates large uncertainties in the optimal values. We have found for the exponent $q$ a mean value of 1.2 and a standard deviation of 0.45 , with $|q-1|<1$ in all cases. This result confirms the assumption $b \gg c$, validating the second-order expansion of $\left\langle\dot{x}_{i} / x_{i}\right\rangle$. Moreover, as seen in Fig. 3 (our fourth result), nice fits are found in general, with very few exceptions.

With this last hypothesis our complete dynamic equation turns out to be

$$
\dot{x}_{i}(t)=k_{q i}(t)\left[x_{i}(t)\right]^{q}+k_{1 i}(t) x_{i}(t)+k_{\frac{1}{2} i}(t) \sqrt{x_{i}(t)},
$$

with $k_{q i}(t), k_{1 i}(t)$ and $k_{\frac{1}{2} i}(t)$ independent Wiener processes. Summing up, we have assumed

(a) a finite-size term that dominates for low population levels $(<1500)$,

(b) a multiplicative term that accounts for population's growth/diminution (births, death, or external migration, and

(c) a power-law (exponent $q \sim 1$ but $q \neq 1$ ) accounting for internal migration.

\section{FROM MICROSCOPIC TO MACROSCOPIC DESCRIPTIONS}

We arrive to stage (iv), having discussed above a microscopic population dynamics. We try now to ascertain whether a macroscopic description is also feasible. Our goal is to reduce the $2 n$ microscopic degrees of freedom [ $\mathbf{x}(t)$ and $\dot{\mathbf{x}}(t)]$ to a few macroscopic ones. We will separately consider each of the three terms of the dynamic equation. The ensuing results will be valid in the domains in which each term dominates.

Consider $n$ random walkers characterized by a dynamic coordinate $x_{i}(t)$ obeying

$$
\dot{x}_{i}(t)=k_{i}(t)\left[x_{i}(t)\right]^{q},
$$

with $\left\langle\left(k_{i}(t)-\bar{k}\right)\left(k_{j}(t)-\bar{k}\right)\right\rangle=\sigma_{k} \delta_{i j} \delta\left(t-t^{\prime}\right)$. Parameter $q$ will take as special possible values $1 / 2$ or 1 , or in general, $0 \leqslant q$. The observed diffusion pattern is expected to depend on the value of $q$. We first explore in analytical detail the case of free diffusion, and secondly we add adequate constraints so as to find stationary states (this issue is also studied in Ref. [34]).

\section{A. Brownian motion and diffusion equation}

We start with $q=0$ as our control case. One has $\dot{x}_{i}(t)=$ $k_{i}(t)$ so that we deal with the well-known Brownian random walkers. Consider this numerical procedure: initially, the $n$ walkers are located at, say, $x=x(0) . \rho(x, t) d x$ will refer to the walkers' normalized histogram, at time $t$, which indicates the (relative) number of walkers positioned within the interval $d x$ around $x$. The associated initial density would read $\rho(x, 0)=\delta[x-x(0)]$. A discrete version of the pertinent dynamic equation is

$$
x_{i}(t+\Delta t)=x_{i}(t)+\Delta t k_{i}(t),
$$

which forces the walkers to "move" during the period $\Delta t$ in a amount given by $\Delta t k_{i}(t)$, with $k_{i}(t)$ a random number generated from a Gaussian distribution determined by a standard deviation $\sigma_{k}$ and mean $\bar{k}$, as defined above. We have at $t=M \Delta t$

$$
x_{i}(t=M \Delta t)=x(0)+\Delta t \sum_{m=1}^{M} k_{i}[(M-1) \Delta t],
$$




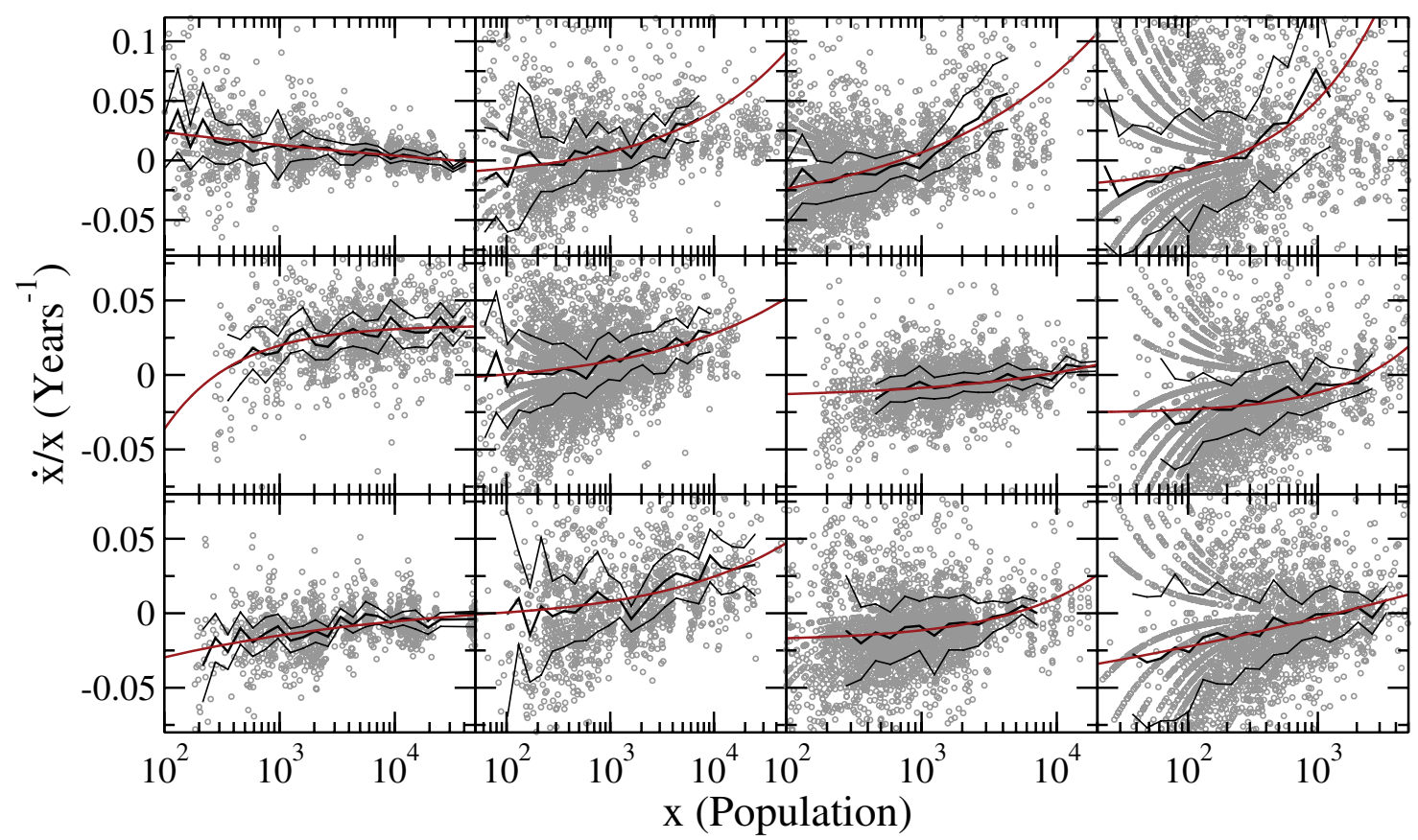

FIG. 3. (Color online) $\dot{x} / x$ vs population (see text) for 12 Spanish provinces (each dot represents one municipality per year). The mean $\langle\dot{x} / x\rangle$, and the limits of the standard deviation as functions of the population, are drawn with black lines. The fit to an expression of the type represented by Eq. (17) is displayed using red lines. The results correspond, respectively, to: Asturias, Almería, Cáceres, Cuenca, Baleares, Lleida, Badajoz, Ávila, Guipúzcoa, Castellón, Valladolid, and Guadalajara.

i.e., after $M$ iterations the walkers' distributions coincides with that of a random number generated by summing up $M$ Gaussian numbers characterized by $\Delta t \sigma_{k}$ and $\Delta t \bar{k}$. Remember that a distribution that follows a random number composed of two other numbers of that character is the convolution of the distributions associated to these later numbers. Thus, $x(t)$ is described by the $M$ th convolution of the $k$ 's Gaussian distribution. By recourse to a Fourier transform $\mathcal{F}$ for convolutions, and setting $x(0)=0$ with no loss of generality, we have

$$
\begin{aligned}
\mathcal{F}[\rho(x, t)] & =\left(\mathcal{F}\left[\frac{e^{-(x-\Delta t \bar{k})^{2} / 2\left(\Delta t \sigma_{k}\right)^{2}}}{\sqrt{2 \pi} \Delta t \sigma_{k}}\right]\right)^{M} \\
& =\left(e^{-\Delta t^{2} \sigma_{k}^{2} \omega^{2} / 2+i \Delta t \bar{k} \omega}\right)^{M} \\
& =e^{-M \Delta t^{2} \sigma_{k}^{2} \omega^{2} / 2+i M \Delta t \bar{k} \omega},
\end{aligned}
$$

where $\omega$ is the frequency variable. Appealing now to the inverse transformation leads to

$$
\begin{aligned}
\rho(x, t) & =\frac{e^{-(x-M \Delta t \bar{k})^{2} /\left(2 M\left(\Delta t \sigma_{k}\right)^{2}\right)}}{\sqrt{2 \pi M} \Delta t \sigma_{k}} \\
& =\frac{e^{-(x-t \bar{k})^{2} /(4 D t)}}{\sqrt{4 \pi D t}},
\end{aligned}
$$

where we have introduced for convenience $2 D=\Delta t \sigma_{k}^{2}$. An arbitrary density $\rho(x, t)$ will evolve in $\Delta t$, via the convolution of that density with a Gaussian of deviation $\Delta t \sigma_{k}=\sqrt{2 \Delta t D}$ and mean $\Delta t \bar{k}$, as

$$
\begin{aligned}
\mathcal{F}[\rho(x, t+\Delta t)] & =\mathcal{F}[\rho(x, t)] e^{-\Delta t D \omega^{2}+i \Delta t \bar{k} \omega} \\
& \simeq \mathcal{F}[\rho(x, t)]\left(1-\Delta t D \omega^{2}+i \Delta t \bar{k} \omega\right),
\end{aligned}
$$

where we take $\Delta t$ arbitrarily small. A simple manipulation involving division by $\Delta t$ leads now to

$$
\frac{\mathcal{F}[\rho(x, t+\Delta t)]-\mathcal{F}[\rho(x, t)]}{\Delta t}=\left(-D \omega^{2}+i \bar{k} \omega\right) \mathcal{F}[\rho(x, t)] .
$$

By recourse again to the inverse transformation and taking the limit $\Delta t \rightarrow 0$ we get

$$
\partial_{t} \rho(x, t)=D \partial_{x}^{2} \rho(x, t)-\bar{k} \partial_{x} \rho(x, t),
$$

which is a diffusion equation. Accordingly, we reach an important result here (our fifth one): Our original $2 n$ degrees of freedom problem can now be tackled via just a few macroscopic parameters.

\section{B. $q$-metric Brownian motion}

We deal now with the general instance $q \neq 0$. Following the recipe of Refs. [33,34], we define first the generalized functions,

$$
\begin{aligned}
\log _{q}(x) & =\frac{x^{1-q}-1}{1-q} ; \quad(x>0), \\
e_{q}(v) & =[1+(1-q) v]_{+}^{1 /(1-q)},
\end{aligned}
$$

also known as Tsallis's $q$ logarithm and $q$ exponential, respectively [38] (by definition $\log _{1}(x) \equiv \ln (x)$ and $e_{1}(v) \equiv e^{v}$ ). We use them to introduce a new variable $u_{i}=\log _{q}\left(x_{i}\right)$. The Jacobian of the transform is $d u / d x=1 / x^{q}$ so that $\dot{u}=\dot{x} / x^{q}$, and the associated dynamical equation Eq. (20) becomes

$$
\dot{u}_{i}(t)=k_{i}(t) .
$$


In the set $\left\{\left(u_{i}, \dot{u}_{i}\right)\right\}_{i=1}^{n}$, the variables $u_{i}$ and $\dot{u}_{i}$ are now independent of each other. We regard them, of course, as our dynamical variables. Note that we have transformed the initial $q$ symmetry of Eq. (20) into a translational one, recovering the well-known Brownian motion for the variable $u$. Indeed,

$$
u_{i}(t=M \Delta t)=u_{i}(0)+\Delta t \sum_{m=1}^{M} k_{i}[(M-1) \Delta t]
$$

and then the demonstration of the preceding subsection becomes valid, now for $u$ and $\rho(u, t) d u$. Our new diffusion equation reads

$$
\partial_{t} \rho(u, t)=D \partial_{u}^{2} \rho(u, t)-\bar{k} \partial_{u} \rho(u, t),
$$

and, starting from a density $\rho(u, 0)=\delta\left(u-u_{0}\right)$ we end up with

$$
\rho(u, t) d u=\frac{d u}{4 \pi D t} \exp \left[-\frac{\left(u-u_{0}-\bar{k} t\right)^{2}}{4 D t}\right] .
$$

The $x$ density is, accordingly, governed by a $q$ log-normal distribution

$$
\begin{aligned}
\rho_{X}(x, t) d x & =\rho[u(x), t] \frac{d x}{d u} d u \\
& =\frac{d x}{\sqrt{4 \pi D t} x^{q}} \exp \left[-\frac{\left[\log _{q}(x)-u_{0}-\bar{k} t\right]^{2}}{4 D t}\right] .
\end{aligned}
$$

In particular, for $q=1 / 2$ one has

$\rho_{X}(x, t) d x=\frac{d x}{\sqrt{4 \pi D t x}} \exp \left[-\frac{\left[2(\sqrt{x}-1)-u_{0}-\bar{k} t\right]^{2}}{4 D t}\right]$,

and, for $q=1$ the well-known log-normal

$$
\rho_{X}(x, t) d x=\frac{d x}{\sqrt{4 \pi D t} x} \exp \left[-\frac{\left[\ln (x)-u_{0}-\bar{k} t\right]^{2}}{4 D t}\right] .
$$

We have again reduced the large number of microscopic degrees of freedom to just a few macroscopic parameters.

\section{Examples of diffusion}

Numerical experiments confirm our findings above. We start with our dynamical equation in discrete form

$$
x_{i}(t+\Delta t)=x_{i}(t)+\Delta t k_{i}(t)\left[x_{i}(t)\right]^{q}
$$

using $k_{i}(t)=\sqrt{2 D / \Delta t} \xi_{i}(t)+\bar{k}$, where the random numbers $\xi$ follow a normal distribution such that $\left\langle\xi_{i}(t) \xi_{j}(t)\right\rangle=\delta_{i j} \delta(t-$ $\left.t^{\prime}\right)$. We have taken $q=1 / 2$ and 1 for our examples, and find that the associated distributions exactly follow the diffusion equation's predictions. We have used in the former case $u_{0}=$ $\log _{1 / 2}(220), \bar{k}=0$, and $\sigma_{k}^{2}=10$, in intervals of $\Delta t=0.01$. In the later instance we had $u_{0}=\ln (4400)$ instead. Indeed, the walkers' histograms' evolution follows Eqs. (34) and (35), respectively, with $D=\Delta t \sigma_{k}^{2} / 2$ as defined above [see Fig. 4 for the cumulative distributions $\left.P(x)=\int_{0}^{x} d x^{\prime} \rho\left(x^{\prime}\right)\right]$.

As empirical examples we discovered that for small populations $(<1500$ inhabitants) the finite-size noise dominates.
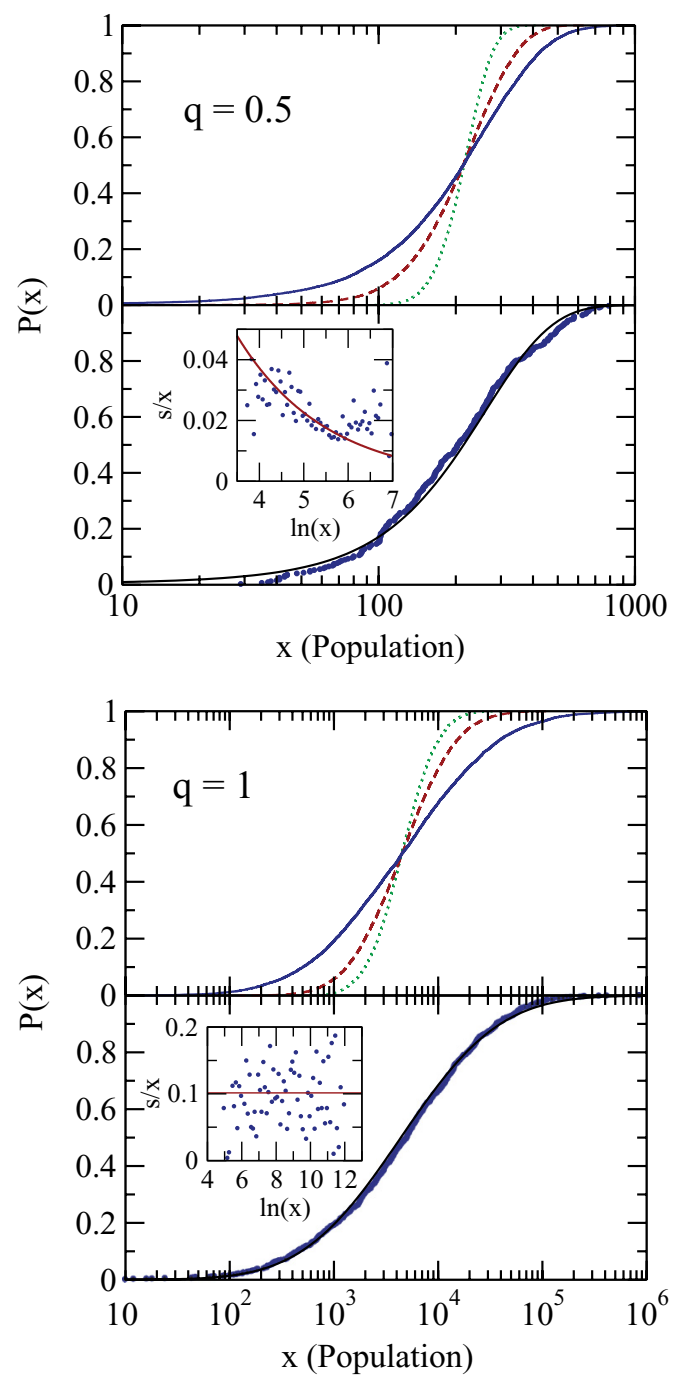

FIG. 4. (Color online) Top: cumulative distribution $P(x)$ at three different times, pertaining to a simulated $q$-metric diffusion process with $q=1 / 2$. We start with the green-dotted distribution. Things evolve and one reaches the red-dashed distribution. Finally, one arrives to the blue-solid configuration. This last distribution is compared with the cumulative distribution of Salamanca's towns' population (blue dots). Inset: variance of the relative growth (in years $^{-1}$ ) vs log population (dots), confirming the $\sqrt{x}$ dependence for $q=1 / 2$ dynamics (red line) (see text). Bottom: geometric diffusion $q=1$ compared with the population distribution of Florida (US). Inset: same as top panel's inset, confirming this time the size independence of our results and thus the proportional nature of the underlying dynamics (see text).

Provinces for which most towns are scarcely populated will obey the dynamical equation with $q=1 / 2$. Such is the case for the province of (i.e., Salamanca) as shown in the top panel of Fig. 4. The ensuing dynamics confirms this assertion. The relative growth of most of the towns follows a dynamics with a variance $s^{2} / x \propto 1 / \sqrt{x}$ (red line of the inset). The ensuing distribution fits the final state predicted by the diffusion equation for that dynamics, Eq. (34), with $u_{0}+\bar{k} t=$ $\log _{1 / 2}(216.3)$ and $2 D t=95.6$ for year 2010 (see Fig. 4). We remark that the 1/2-log-normal can be easily confused with the usual log-normal, although the former exhibits asymmetries in 


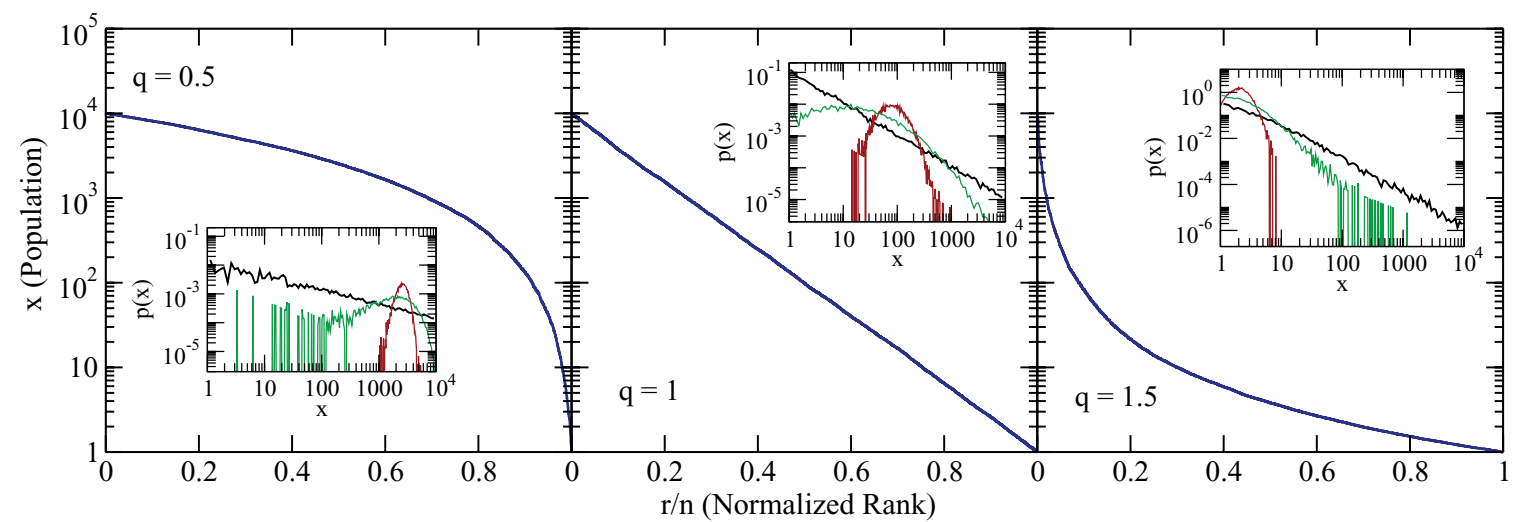

FIG. 5. (Color online) Equilibrium rank distributions for $q$-metric diffusion processes with maximum size constraints for (from left to right), $q=0.5,1,1.5$, and $n=10000$. Insets: evolution of the density distribution of the diffusion process, starting with a $\delta$ configuration in $x$ and passing through the red and green configurations, until equilibrium is attained for the black distribution (see text).

$\log$ scale. As a $q=1$ example we mention the state of Florida in the US [39] (see also bottom Fig. 4). Using data from 1990, 2000, and 2010, we have verified that the microscopic dynamics confirms the proportional growth assumption (with a variance of the relative growth independent of the size, as illustrated in the inset). The city-populations distribution follows a log-normal distribution, that of Eq. (35), which can be the one pertaining to geometrical random-walkers' diffusion, with $u_{0}+\bar{k} t=\ln (4380)$ and $2 D t=2.96$.

\section{Constrained diffusion}

$q$-log-normal distributions do not set any limits to population sizes. However, it is reasonable to assume that physical space does pose limits to a city's population growth. Unlimited growth is unrealistic since in the case of internal migrations the total population $N_{T}$ should remain constant and a freediffusion model is, again, unrealistic. Constrained diffusion must be contemplated instead.

We pass now to consider numerical experiments with random walkers that fix lower and upper bounds for population. These are denoted by $x_{0}$ and $x_{M}$, respectively. Now, walkers' moves leading to values outside the range $x_{0}<x<x_{M}$ are to be rejected in our simulations. Figure 5 shows that a $q$-metric walkers' evolution begins by faithfully following the diffusion equation Eq. (31) until they bump off these extreme values. Now their density deviates from that of "free" evolution. After some time has elapsed, an equilibrium $x$ distribution is reached that follows a power law with exponent $q$, independently of the initial state. The origin of this systematic result can not be unravelled by the simulations, so a higher level of theory is needed.

Now we use a total population constraint. This is equivalent to making the walkers move under the rule of a $q$-generalized multicomponent logistic equation

$$
\dot{x}_{i}(t)=\left[x_{i}(t)\right]^{q}\left[k_{i}(t)-\frac{\sum_{i=1}^{n} k_{i}(t)\left[x_{i}(t)\right]^{q}}{\sum_{i=1}^{n}\left[x_{i}(t)\right]^{q}}\right] .
$$

Indeed, it is easy to check that $\partial_{t} N_{T}=\sum_{i=0}^{n} \dot{x}_{i}(t)=0$, thus preserving the value of $N_{T}$ in time. Also the original $q$ symmetry of the dynamics is preserved. This equation is the $q$ generalization of the scale-invariant multicomponent logistic equation presented in Ref. [35]. Results are displayed in Fig. 6 for $q=0,1$ and 2, using $n=100000$ walkers and a total population of $N=250000$ inhabitants (with $x_{0}=1$ ). Remarkably enough, equilibrium is always reached, to a density that does not depend upon the initial state or the $k$ parameters. The shape of the distributions resembles $x$ power laws with exponential cutoff. Again, the simulation can not unravel the origin of this form. Finding the properties and the exact analytical form of those macroscopic equilibrium distributions is our goal in the sext section.

\section{MACROSCOPIC CONUNDRUM}

We deal now with step (v). Our simulations with random walkers suggest that it is indeed possible to pass from a description that uses $2 n$ microscopic variables to a description involving just a few macroscopic parameters. The big question is: do they behave in thermodynamic fashion, satisfying the pertinent partial derivatives relationships? We wish to tackle this issue now, looking for a way to reduce the number of microscopic degrees of freedom to a few manageable macroscopic ones while keeping a coherent, reasonable description of our system, mimicking the kind of scenario that links statistical mechanics to thermodynamics. This requires appropriate constraints, a topic to be addressed below by enumerating the appropriate "social" constraints we need.

\section{A. Macroscopic constraints}

Total number of cities $n$. Since there is some confusion in the available data about what the administrative meaning of city is, we wish to ascertain that this issue is not of great importance for proportional growth. Consider $x_{i}=\sum_{j}^{n_{i}} x_{i j}$, where $n_{i}$ is the number of sub-administrative units included in the administrative unit $i$, with $x_{i j}$ their subadministrative populations. Considering proportional growth, we write for the time evolution

$$
\begin{aligned}
\dot{x}_{i}(t) & =\sum_{j}^{n_{i}} \dot{x}_{i j}(t)=\sum_{j}^{n_{i}} k_{i j}(t) x_{i j}(t) \\
& =\frac{\sum_{j}^{n_{i}} k_{i j}(t) x_{i j}(t)}{\sum_{j}^{n_{i}} x_{i j}(t)} \sum_{j}^{n_{i}} x_{i j}(t)=k_{i}^{\prime}(t) x_{i}(t),
\end{aligned}
$$



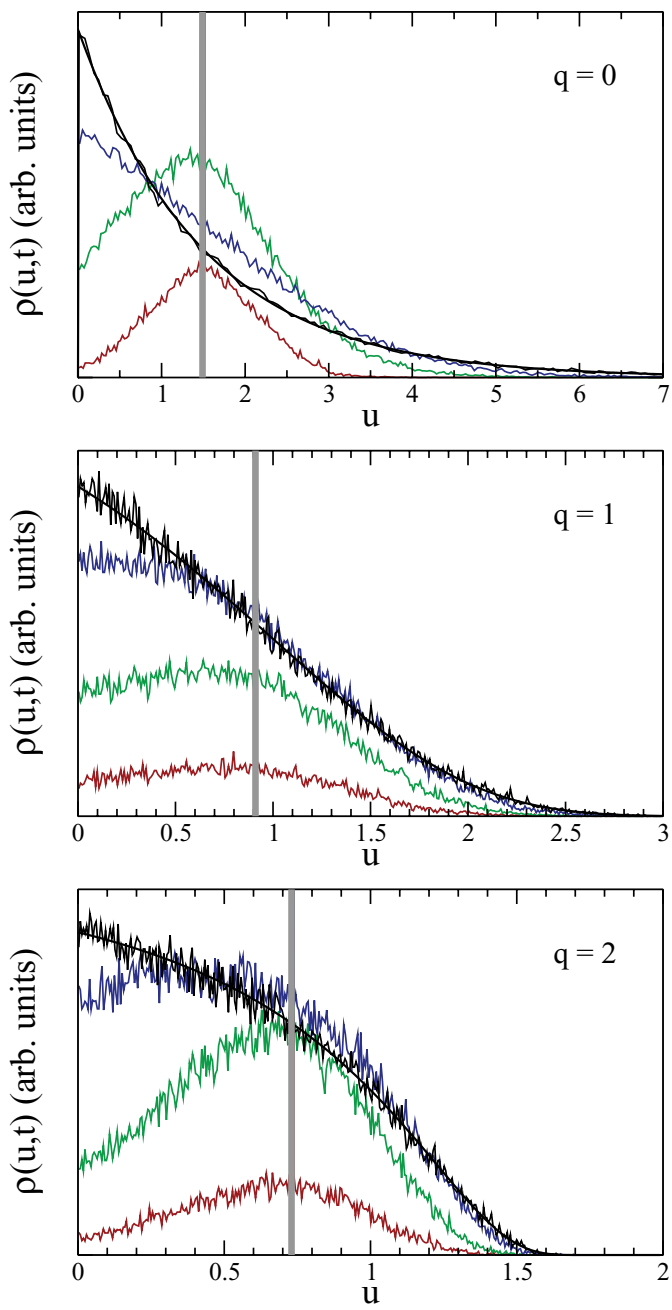

FIG. 6. (Color online) Evolution of the density distribution from an initial $\delta$ state (gray solid bar), passing through intermediate nonequilibrium configurations red-green-blue, until reaching equilibrium (solid black line). We deal with $N$-constrained, $q$-metric diffusion processes for (from top to bottom), $q=0,1$, and 2 . The smooth black lines follow Eq. (73) in $u$ space, for each $q$ value.

where we have defined $k_{i}^{\prime}(t)$ as a new variable defined as an average weighted by the populations $x_{i j}$. If the growth rates $k_{i j}$ are random variables with approximately the same mean and variance, it is easy to check that $k_{i}^{\prime}(t)$ is in turn a random variable of the same mean and variance. The dynamical behavior of the ensemble of administrative units $\mathbf{x}$ is thus equivalent of that of the subunits, and the procedure described in this work is still applicable.

Maximum/minimum population $x_{M} / x_{0}$. It is well known that a typical minimum population size equals the Dunbar number [40] $(\sim 150)$, heuristically associated to the maximum (allowable by our neocortex) number of stable human relationships. Thus, it is reasonable to think of a minimum size $x_{0} \sim 150$. In many cases a maximum number for a city population $x_{M}$ can be established via consideration of geographical peculiarities as mountains [41] or oceans [42] (see Fig. 7 for an example). In such cases it is convenient to employ the transform $u=\log _{q}\left(x / x_{0}\right)$. An associated, valuable macroscopic parameter is $u_{M}=\log _{q}\left(x_{M} / x_{0}\right)$. We will be dealing then with a "volume" $0<u<u_{M}$.

Total population $N_{T}$. We have $N_{T}=\sum_{i=1}^{n} x_{i}$ that gets transformed into $N_{T}=x_{0} \sum_{i=1}^{n} e_{q}^{u_{i}}$. A useful quantity becomes then $N=N_{T} / x_{0}$.

Total variance of $\dot{u}$. With reference to the dynamics, a useful observable is the total variance for relative growth $\sigma^{2}=\sum_{i=1}^{n}\left\langle\left(\dot{u}_{i}-\left\langle\dot{u}_{i}\right\rangle_{t}\right)^{2}\right\rangle / n$. For a Gaussian form (see Fig. 1) this quantity measures fluctuation intensities. Generalizing, this quantity can be defined by the covariance matrix with elements $Q_{i j}=\left\langle\left(\dot{u}_{i}-\left\langle\dot{u}_{i}\right\rangle\right)\left(\dot{u}_{j}-\left\langle\dot{u}_{j}\right\rangle\right)\right\rangle$. We use its trace as a thermodynamical variable $\operatorname{Tr}(Q)=\sum_{i=1}^{n} Q_{i i}$ defining

$$
U=\frac{\tau}{2} \operatorname{Tr}(Q)=\frac{\tau}{2} \sum_{i=1}^{n}\left\langle\left(\dot{u}_{i}-\left\langle\dot{u}_{i}\right\rangle\right)^{2}\right\rangle=\frac{\tau}{2} n \sigma^{2},
$$

where we add for dimensional convenience a factor $\tau / 2$.

\section{B. Fundamental hypothesis for urban thermodynamics}

Let us discuss the three hypothesis that we need in our scheme:

H-I. Microscopic hypothesis. We adopt as fundamental dynamical equation Eq. (20) $\left[\dot{x}=k x^{q}\right]$ for the population of a center, linearized via the variable $u=\log _{q}\left(x / x_{0}\right)$. We will think of the pair $(u, \dot{u})$ as constituting our social phase space coordinates. We can speak of an:

H-II. A priori phase space equiprobability in $(u, \dot{u})$ [37]. The probability density distribution for the $i$ th phase space cell centered at $\left(u_{i}, \dot{u}_{i}\right)$ of size $d u d \dot{u}$ is defined as $\rho\left[\left\{\left(u_{i}, \dot{u}_{i}\right)\right\}_{i=1}^{n}\right] d^{n} u d^{n} \dot{u}$. According to H-II, the system's entropy is written as

$$
S[\rho]=-\int d^{n} u d^{n} \dot{u} \rho\left[\left\{\left(u_{i}, \dot{u}_{i}\right)\right\}_{i=1}^{n}\right] \ln \left[\rho\left[\left\{\left(u_{i}, \dot{u}_{i}\right)\right\}_{i=1}^{n}\right]\right] .
$$

Since none of our macroscopic observables is able to distinguish amongst population nuclei, towns are thus indistinguishable. In this case, the useful distribution is the one-body density $\rho(u, \dot{u})$ defined as

$$
\rho(u, \dot{u})=\int d^{n-1} u d^{n-1} \dot{u} \rho\left[\left\{\left(u_{i}, \dot{u}_{i}\right)\right\}_{i=1}^{n}\right],
$$

and thus,

$$
S[\rho]=-\int d u d \dot{u} \rho(u, \dot{u}) \ln [\rho(u, \dot{u})] .
$$

Macroscopic observables are written in terms of the one-body density as

$$
\begin{gathered}
n=\int d u d \dot{u} \rho(u, \dot{u}), \\
N=\int d u d \dot{u} \rho(u, \dot{u}) e_{q}(u), \\
U=\frac{\tau}{2} \int d u d \dot{u} \rho(u, \dot{u}) \dot{u}^{2} .
\end{gathered}
$$

H-III. Maximum entropy principle (MaxEnt) [37]. Equilibrium is determined via constrained entropic maximization using $n, u_{M}, N$, and $U$. This determines the equilibrium density $\rho(u, \dot{u})$ that is a solution of the entropic variational problem

$$
\delta\{S[\rho]-\beta A[\rho]\}=0,
$$




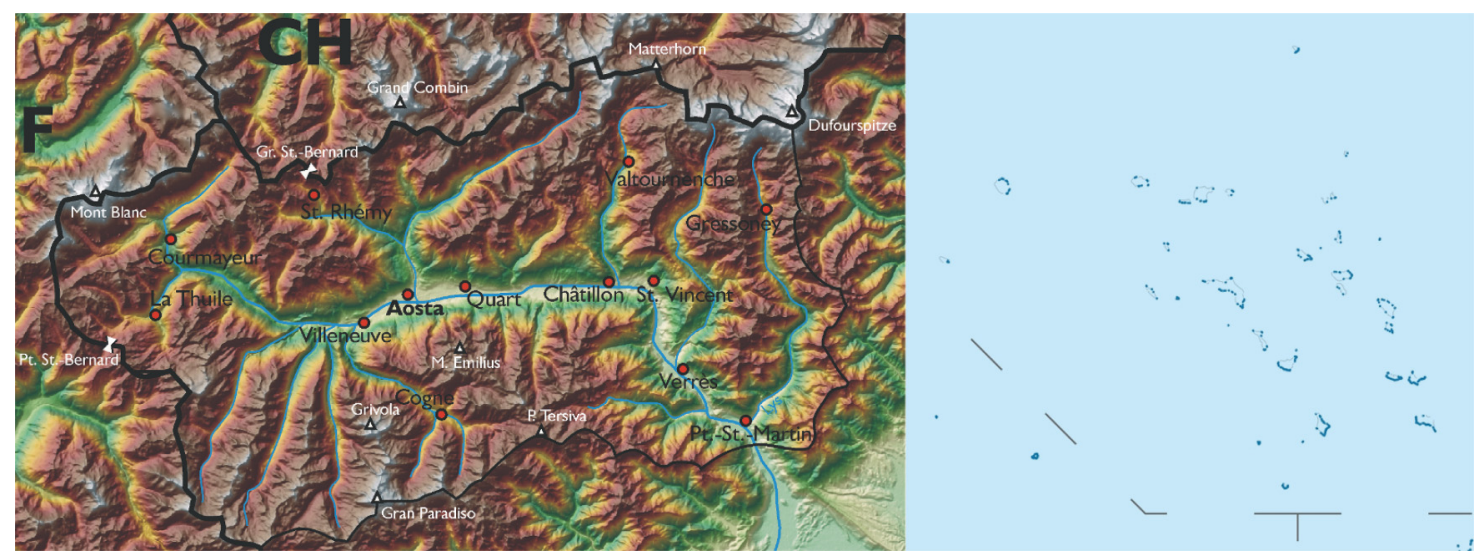

FIG. 7. (Color online) An example of population restriction arising out of geographical reasons, as a mountainous or island landscape. Left: d'Aosta valley (Italy) [41]. Right: Marshall islands [42].

with

$$
A=U-\mu n+p u_{M}+\Lambda N,
$$

where $\beta, \mu, p$ and $\Lambda$ stand for the pertinent Lagrange multipliers, that will be seen below to acquire the character of intensive thermal-quantities.

\section{Thermodynamical relations}

We enter step (vi) by considering the Lagrangian $A[\rho]$ [and Lagrangian density $a(u, \dot{u})]$. It reads

$$
\begin{aligned}
A[\rho] & =\int d u d \dot{u} \rho(u, \dot{u}) a(u, \dot{u}) \\
& =\int d u d \dot{u} \rho(u, \dot{u})\left\{\frac{\tau}{2} \dot{u}^{2}-\mu+p v(u)+\Lambda e_{q}(u)\right\},
\end{aligned}
$$

where the volume condition is enforced by an infinite-well potential

$$
v(u)= \begin{cases}u_{M} / n & \text { for } 0<u<u_{M} \\ \infty & \text { otherwise }\end{cases}
$$

The well-known general solution to the entropic problem Eq. (46) is [37] $\rho(u, \dot{u})=\exp [-\beta a(u, \dot{u})]$, so that

$$
\rho(u, \dot{u})=\frac{n}{Z} e^{-\frac{\beta \tau}{2} \dot{u}^{2}-\beta \Lambda e_{q}(u)}\left(0<u<u_{M}\right),
$$

where the normalization factor $Z$ (partition function) becomes

$$
\begin{aligned}
Z & =\int_{-\infty}^{\infty} d \dot{u} \int_{0}^{u_{M}} d u e^{-\frac{\beta \tau}{2} \dot{u}^{2}-\beta \Lambda e_{q}(u)} \\
& =\sqrt{\frac{2 \pi}{\beta \tau}} E_{q}\left(\beta \Lambda, u_{M}\right),
\end{aligned}
$$

with $E_{q}(l, m)$ defined as

$$
E_{q}(l, m)=E_{q}(l)-e^{(1-q) m} E_{q}\left(l e^{m}\right),
$$

where $E_{q}(l)$ is the exponential function of order $q$. Our constraints in $U$ and $N$ determine the multipliers $\beta$ and $\Lambda$ values. Indeed, on the one hand, we have the $\dot{u}$ variance

$$
U=\frac{\tau}{2} n \int_{-\infty}^{\infty} d \dot{u} \sqrt{\frac{\beta \tau}{2 \pi}} e^{-\frac{\beta \tau}{2} \dot{u}^{2}} \dot{u}^{2}=\frac{n}{2 \beta},
$$

and on the other hand, we deal with the total population

$$
\begin{aligned}
N & =n \int_{0}^{u_{M}} d u \frac{e_{q}(u) e^{-\beta \Lambda e_{q}(u)}}{E_{q}\left(\beta \Lambda, u_{M}\right)} \\
& =n \frac{E_{q-1}\left(\beta \Lambda, u_{M}\right)}{E_{q}\left(\beta \Lambda, u_{M}\right)}=n F_{q}\left(\beta \Lambda, u_{M}\right),
\end{aligned}
$$

where we use the function $F_{q}(l, m)=\partial_{l} \ln \left[E_{q}(l, m)\right]$. We obtain from the former the direct result

$$
\beta=\frac{n}{2 U},
$$

and, via inversion of the latter equation [defining first $L_{q}(f, m)=F_{q}^{-1}(f, m)$ and thus $\left.F_{q}\left[L_{q}(f, m), m\right]=f\right]$, we finally obtain the relation between the system variables (equation of state)

$$
\beta \Lambda=L_{q}\left(N / n, u_{M}\right) .
$$

Note that we have intensive quantities on the left-hand side, while extensive ones appear in the right-hand side. The entropy becomes

$$
\begin{aligned}
S=n \ln & {\left[\frac{1}{n} \sqrt{\frac{2 \pi}{\beta \tau}} E_{q}\left(\beta \Lambda, u_{M}\right)\right] } \\
+n & {\left[\frac{1}{2}+\beta \Lambda F_{q}\left(\beta \Lambda, u_{M}\right)\right] . }
\end{aligned}
$$

Using now Eq. (56) we can recast things in term of the natural variables as

$$
\begin{aligned}
S\left(U, n, u_{M}, N\right)= & n \ln \left[\frac{2}{n} \sqrt{\frac{\pi U}{n \tau}} E_{q}\left[L_{q}\left(N / n, u_{M}\right), u_{M}\right]\right] \\
& +\frac{n}{2}+L_{q}\left(N / n, u_{M}\right) N .
\end{aligned}
$$

It is easy to verify, but crucial to our present goals, that macroscopic observables and Lagrange multipliers become linked entropicwise via

$$
\begin{gathered}
\beta=\left.\frac{\partial S}{\partial U}\right|_{n, u_{M}, N}, \\
\mu=\left.\frac{1}{\beta} \frac{\partial S}{\partial n}\right|_{U, u_{M}, N},
\end{gathered}
$$




$$
\begin{gathered}
p=\left.\frac{1}{\beta} \frac{\partial S}{\partial u_{M}}\right|_{U, n, N}, \\
\Lambda=\left.\frac{1}{\beta} \frac{\partial S}{\partial N}\right|_{U, u_{M}, n} .
\end{gathered}
$$

The first relation leads to Eq. (55), also showing that the $\beta$ multiplier is the inverse of the variance $\beta=1 / \tau \sigma^{2}$. The last relation takes us to Eq. (56) and is indeed one of our equations of state. The other two are

$$
\begin{aligned}
\beta \mu= & \ln \left[\frac{2}{n} \sqrt{\frac{\pi U}{n \tau}} E_{q}\left[L_{q}\left(N / n, u_{M}\right), u_{M}\right]\right]-1 \\
& -2 L_{q}^{(1,0)}\left(N / n, u_{M}\right)\left(\frac{N}{n}\right)^{2}
\end{aligned}
$$

and

$$
\begin{aligned}
\beta p= & n \frac{\exp \left[-L_{q}\left(N / n, u_{M}\right) e_{q}\left(u_{M}\right)\right]}{E_{q}\left[L_{q}\left(N / n, u_{M}\right), u_{M}\right]} \\
& +2 L_{q}^{(0,1)}\left(N / n, u_{M}\right) N .
\end{aligned}
$$

where the superscripts label the argument which is being differentiated.

At this stage the reader will agree that it is fair to assert that our goal has been successfully reached. We have indeed constructed a social thermodynamics for urban population flows.

\section{APPLICATION: SCALE-FREE IDEAL GAS (SFIG)}

This is our final step (vii), where we apply our formalism to empirical systems. We envision two main regimes, according to the $\Lambda$ value: $\Lambda=0$ and $\Lambda>0$.

\section{A. SFIG-in-a-box}

We will consider in some detail the first case here. Different scenarios can be associated to $\Lambda \rightarrow 0$ : (i) the system is not isolated and exchanges population with its surroundings, with a maximum-size constraint, (ii) the triplet $n, N, u_{M}$ is such that the equation of state yields $\Lambda=0$ (i.e., $N / n=$ $\left.\log _{q-1}\left[e_{q}\left(u_{M}\right)\right] / u_{M}\right)$, or (iii) no size limitation exists $\left(u_{M} \rightarrow\right.$ $\infty)$ but $N / n$ is large enough so that $\Lambda \sim 0$. In the latter case one can obtain an effective $u_{M}$ value from normalization such that $u_{M}=E_{q}(\beta \Lambda)$. When $\Lambda=0$ the Lagrangian $A$ is written as

$$
A=U-\mu n+p u_{M}
$$

so that we do not need knowledge of $N$. The equilibrium density is

$$
\rho(u, \dot{u}) d u d \dot{u}=\frac{n}{u_{M}} \sqrt{\frac{\beta \tau}{2 \pi}} e^{-\frac{\beta \tau}{2} \dot{u}^{2}} d u d \dot{u}\left(0<u<u_{M}\right) .
$$

The partial density $\rho(u)=\int d \dot{u} \rho(u, \dot{u})=n / u_{M}$ is constant in $u$ so that $x$ is given by a power law

$$
\rho_{X}(x) d x=\rho[u(x)] \frac{d u}{d x} d x=n \frac{x_{0}^{q-1}}{u_{M}} \frac{d x}{x^{q}},
$$

with an associated rank plot given by

$$
x(r)=x_{0} e_{q}\left[u_{M}(1-r / n)\right],
$$

TABLE I. Macroscopic parameters for the four SFIG-in-a-box examples $\left(\tau=100\right.$ years $\left.^{2}\right)$.

\begin{tabular}{lccc}
\hline \hline & Marshall Islands & Agosta Valley & Huelva \\
\hline$n$ & 160 & 74 & 79 \\
$x_{0}$ & 2.14 & 126 & 206 \\
$u_{M}$ & 0.038 & 0.048 & 0.059 \\
$\beta$ & 0.504 & 2.05 & 11.7 \\
$p$ & 0.829 & 0.0133 & 0.0115 \\
\hline \hline
\end{tabular}

where $r$ is the rank from 1 to $n$. Comparing with the equilibrium densities found above in our numerical experiments with random walkers, a very nice fit ensues as seen in Fig. 5, which validates our methodology. The entropy becomes

$$
S\left(U, n, u_{M}\right)=n \ln \left[\frac{2}{n} \sqrt{\frac{\pi U}{n \tau}} u_{M}\right]+\frac{n}{2},
$$

resembling that of the one-dimensional ideal gas. The state equations are

$$
\beta \mu=\ln \left[\frac{2}{n} \sqrt{\frac{\pi U}{n \tau}} u_{M}\right]-1
$$

and

$$
\beta p=\frac{n}{u_{M}},
$$

in exact agreement with the ideal gas scenario.

As empirical $q=1$ examples we discuss the cases of (i) Marshall Islands [43], (ii) d'Agosta Valley (Italy) [44], and (iii) Huelva province (Spain) [36] (Fig. 8). In all instances, the relative growth is nearly independent of the population. Remarkably, low-populated cities can (i) follow the general trend, as in Marshall Islands, (ii) insinuate a secondary constant trend, as for d'Agosta Valley, or (iii) be affected by finite-size noise, as in the case of Huelva. Thus, we consider that the overall microscopic dynamics fits the proportional growth hypothesis, with densities $\rho(u, \dot{u})$ nicely adapted to the ensuing thermodynamic predictions. In all cases, geographical conditions set strong limits to the city sizes. The values for the macroscopic parameters are shown in Table I. Remarkably enough, the pressure $p$ due to the limited space is highest for the Marshall Islands. Indeed, this system exhibits the lowest volume $u_{M}$ and the lowest $\beta$ (highest "temperature") for a large number of units $n$.

\section{B. SFIG under total population constraint}

We now consider $\Lambda>0$, with $u_{M} \rightarrow \infty$ for simplicity. This case describes regions where internal migration dominates the microscopic dynamics, and no upper limit is encountered for the city size. This situation can be found when the total population changes relatively slowly in comparison with the fluctuations of cities' population (i.e., the change in $N$ is of a quasistatic nature). According to the equation of state (64), one has $p=0$ in this limit, so that we deal with the Lagrangian

$$
A=U-\mu n+\Lambda N .
$$



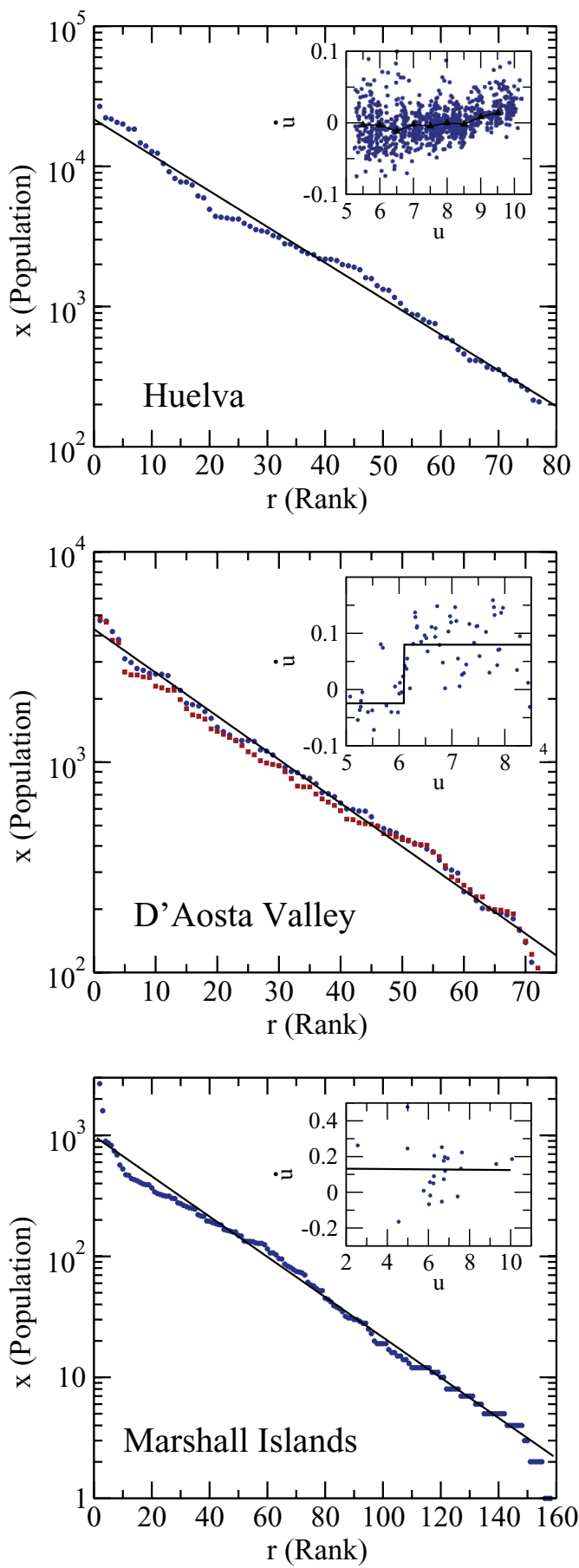

FIG. 8. (Color online) SFIG-in-a-box examples, from top to bottom: rank distribution of Huelva province, Spain (2010), D'Aosta Valley, Italy (2000 in red and 2010 in blue), and Marshall Islands (2010), compared with distribution (68), plotted as a continuous curve. In the insets, the relative growth $\dot{u}$ in years ${ }^{-1}$ vs the log population $u$.

The equilibrium density is

$$
\rho(u, \dot{u}) d u d \dot{u}=\frac{n}{E_{q}(\beta \Lambda)} \sqrt{\frac{\beta \tau}{2 \pi}} e^{-\frac{\beta \tau}{2} \dot{u}^{2}-\beta \Lambda e_{q}(u)} d u d \dot{u}(0<u) .
$$

TABLE II. Macroscopic parameters for the SFIG-underpopulation-constraint examples $\left(\tau=100\right.$ years $\left.^{2}\right)$.

\begin{tabular}{lcccc}
\hline \hline & Alicante & Almería & Girona & Lleida \\
\hline$n$ & 140 & 101 & 220 & 230 \\
$x_{0}$ & 83.9 & 147 & 141 & 105 \\
$N$ & 18355.7 & 3245.53 & 4597.37 & 2818.12 \\
$\ln (\beta \Lambda)$ & -6.26 & -5.35 & -5.18 & -4.02 \\
$\Lambda$ & $4.0310^{-3}$ & $1.8310^{-4}$ & $7.8910^{-5}$ & $4.110^{-4}$ \\
$\beta$ & 0.47 & 25.9 & 71.1 & 43.8 \\
$q$ & 0.862 & 1.135 & 1.27 & 1.16 \\
& Navarra & Vizcaya & Zaragoza & Granada \\
$n$ & 271 & 111 & 292 & 167 \\
$x_{0}$ & 47.6 & 219 & 40.7 & 263 \\
$N$ & 8976.55 & 3571.01 & 6906.03 & 2539.5 \\
$\ln (\beta \Lambda)$ & -5.16 & -5.18 & -5.06 & -4.01 \\
$\Lambda$ & $6.6910^{-4}$ & $1.1810^{-4}$ & $6.2210^{-4}$ & $2.0310^{-3}$ \\
$\beta$ & 8.57 & 47.4 & 10.2 & 8.91 \\
$q$ & 1.06 & 1.08 & 1.18 & 1.02 \\
\hline \hline
\end{tabular}

The partial density for $x$ is given by a power law with exponential cutoff

$$
\rho_{X}(x) d x=n \frac{x_{0}^{q-1}}{E_{q}(\beta \Lambda)} \frac{e^{-\Lambda x}}{x^{q}} d x,
$$

with an associated rank plot

$$
x(r)=\frac{x_{0}}{\Lambda} E_{q}^{-1}\left[E_{q}(\Lambda) r / n\right] .
$$

Again, this result fits the numerical equilibrium densities found above in our numerical simulations (Fig. 6), validating again our methodology.

This is a typical situation for the Spanish provinces (more details are given in Ref. [33]). We have found a nice agreement between (i) the $q$ value obtained from a fit to the microscopic dynamics and (ii) the $q$ value obtained from the fit of the rank plot to Eq. (75). We show some examples in Fig. 9, and the associated macroscopic numerical results in Table II. In the examples presented below, using the parameter $\Lambda$ as a measure of the pressure generated by the total population constraint, it turns out that Alicante is the province with the highest pressure and Girona that with the lowest one, correlated with a highest and a lowest temperature, respectively.

\section{DISCUSSION}

We have shown here how an explicit thermodynamics for population flows can be built from scratch in a data-driven fashion (i.e., proposing hypothesis and validating them with empirical data). We have parameterized first the microscopic dynamics (H-I) by choosing a functional form that best fits the empirical observations. The general form Eq. (20) is a power law and thus entails nonlinear dynamics that loses translational symmetry. However, we present a transformation of variables that linearizes the dynamical equation [Eq. (29)]. The result is that traditional diffusion can be described with the new set of variables $(u, \dot{u})$. We use them to define our phase space, and 


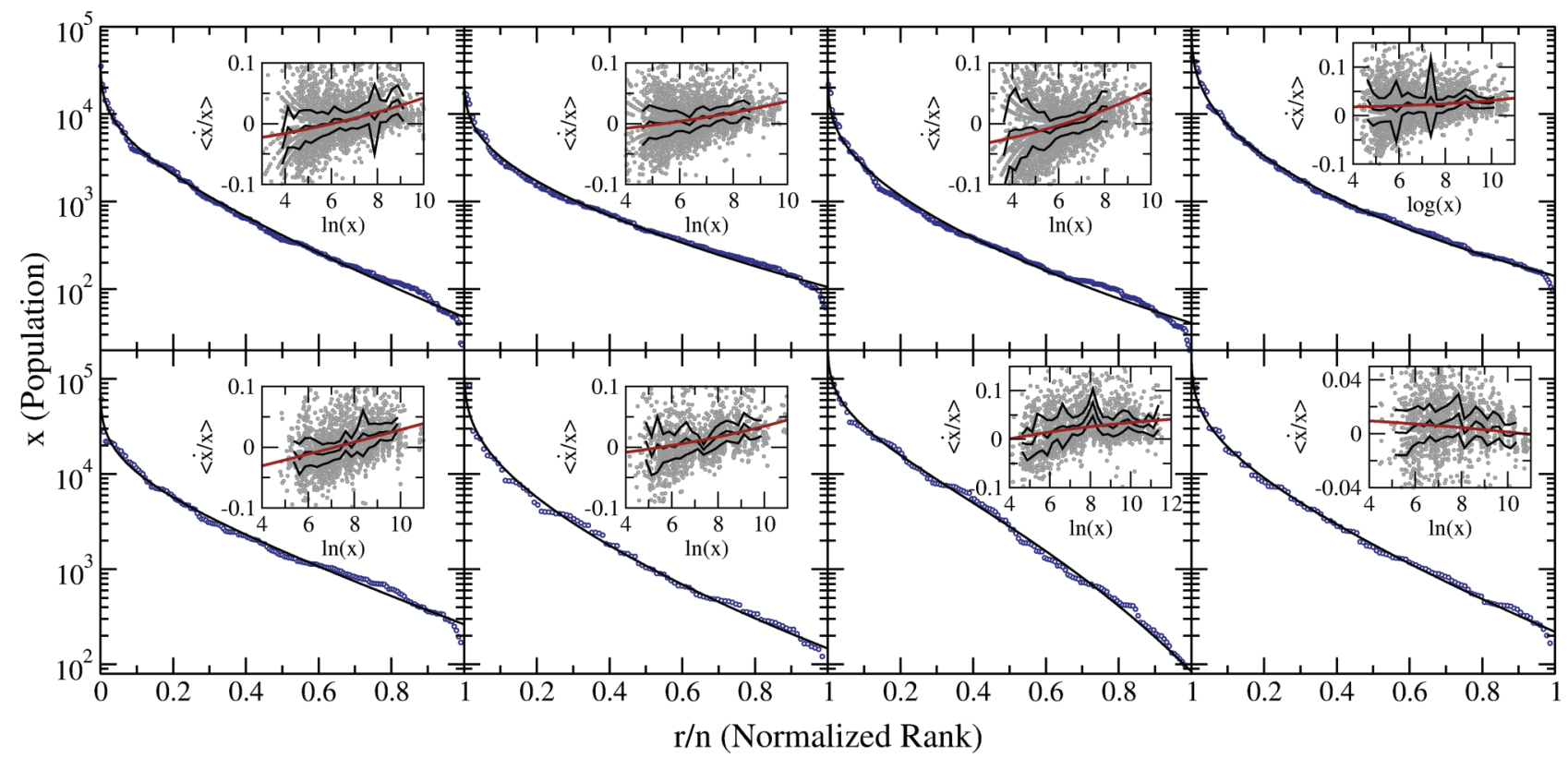

FIG. 9. (Color online) Empirical rank distribution of SFIGs under total population constraint. These examples refer, respectively, to: Navarra, Lleida, Zaragoza, Girona, Granada, Almería, Alicante, and Vizcaya (dots) compared with Eq. (75) (continuous lines). Insets: relative growth $\dot{x} / x$ in years $^{-1}$ vs $\log$ population $\ln (x)$, fitted to Eq. (17) with the same value of $q$ used for the rank distribution.

formulate an a priori probability hypothesis (H-II) for it. An entropy measure can be adequately defined so that the MaxEnt principle (H-III) can be appealed to. Given the principle, a new thermodynamics can be formulated if objective macroscopic observables are well defined. However, we need still to address some further questions below.

\section{A. Potential correlations of the noise}

We have assumed in our derivation that all noises involved are (i) Gaussian and (ii) $\delta$ correlated. Although (i) is well motivated (see Fig. 1), point (ii) has been assumed just for the sake of simplicity. Disregarding correlations leads us to formulate the ideal gas scenario and equations of state can be straightforwardly derived. However, we should expect (i) some level of correlation between neighboring cities and (ii) also some kind of memory, or time correlation. Correlations entail the existence of a nondiagonal covariance matrix $Q$. Since our macroscopic observable is the trace of this matrix, invariant under a basis transformation, the observable $U$ can be expressed in the eigenbasis of $Q$ that diagonalizes this matrix. Thus, the macroscopic observables do not change and our formalism remains applicable. Time correlations in physics usually appear in the guise of inertia. In such case, instead of a dynamical equation as (20), a Langevin-like equation should be formulated to extend our formalism, in analogy to statistical physics. We have studied this phenomena elsewhere (see Ref. [45]).

\section{B. Foundation/abandonment of cities}

We have assumed in this work that the number of settlements remains constant. Indeed, we fix a minimum population $x_{0}$ that prevents the total abandonment of a city (to zero population). The abandonment of a city, or its disappearance as an autonomous administrative unit, is something statistically rare: in 15 years, less than 10 municipalities have been created/deleted in Spain, which is less that a $0.12 \%$ of the total number of municipalities (8116). However, there were some historical periods in which the creation of new settlements was something crucial, as in America or Australia. A model able to describe these phenomena would be indeed very useful for disciplines as quantitative history. Since we have formulated a thermodynamics, we can appeal to the theory of ensembles. The macrocanonical ensemble of urban population flows, in which cities can appear and disappear, is the ideal framework for such models.

\section{Out of equilibrium}

All results presented here (with the exception of those for diffusion) correspond to system in equilibrium. Although some social systems might be in equilibrium, or at least suffer a quasi-static evolution, not all of them will. However, knowing the statistical properties at equilibrium allows one to easily detect nonequilibrium by comparison. Formulation of a nonequilibrium thermodynamics of social phenomena constitutes a formidable challenge.

\section{Other applications}

We have applied our theoretical formulation to city population, but it is expected that other social systems based on groups of people might be also tractable using our formalism. In Ref. [17] both city-population distributions and electoral results were studied using the same procedure. Large data sets are also available for electoral results, so that the hypothesis presented here may be, accordingly, verified and/or modified. Work along such lines is in progress. 


\section{SUMMARY}

After initially introducing some useful social-macroscopic and social-stochastic quantities we have:

(i) Postulated social, dynamic microscopic equations.

(ii) Validated them using urban population data.

(iii) Performed numerical simulations with random walkers that conclusively demonstrated that a description using many microscopic variables has as a counterpart a macroscopic one with few parameters.

(iv) Showed that such macroscopic description can be given an appropriate maximum-entropy-principle's form after constructing a social phase space, which allows one to derive thermodynamiclike relations amongst our macroparameters.

(v) Finally, as an application, we successfully analyzed urban flows as modeled by a scale-invariant ideal gas.

\section{ACKNOWLEDGMENTS}

We appreciate the comments of the reviewers. This work was partially supported by ANR DYNHELIUM (ANR-08BLAN-0146-01) Toulouse, and the Projects PIP1177 of CONICET (Argentina), FIS2008-00781/FIS (MICINN), and FEDER (EU) (Spain, EU).
[1] J. Kemeny and J. L. Snell, Mathematical Models in the Social Sciences (MIT Press, Cambridge, 1978); M. Schroeder, Fractals, chaos and power laws (Freeman, New York, 1990).

[2] M. E. J. Newman, Phys. Rev. E 64, 016131 (2001).

[3] A. Hernando, D. Puigdomènech, D. Villuendas, C. Vesperinas, and A. Plastino, Phys. Lett. A 374, 18 (2009).

[4] A.-L. Barabasi and R. Albert, Rev. Mod. Phys. 74, 47 (2002).

[5] T. Maillart, D. Sornette, S. Spaeth, and G. von Krogh, Phys. Rev. Lett. 101, 218701 (2008).

[6] B. Blasius and R. Tönjes, Phys. Rev. Lett. 103, 218701 (2009).

[7] R. N. Costa Filho, M. P. Almeida, J. S. Andrade, and J. E. Moreira, Phys. Rev. E 60, 1067 (1999).

[8] A. A. Moreira, D. R. Paula, R. N. Costa Filho, and J. S. Andrade, Phys. Rev. E 73, 065101(R) (2006).

[9] L. C. Malacarne, R. S. Mendes, and E. K. Lenzi, Phys. Rev. E 65, 017106 (2001).

[10] M. Marsili and Y. C. Zhang, Phys. Rev. Lett. 80, 2741 (1998).

[11] R. L. Axtell, Science 293, 1818 (2001).

[12] G. K. Zipf, Human Behavior and the Principle of Least Effort (Addison-Wesley, Cambridge, 1949).

[13] Y. Chen, Chaos, Solitons \& Fractals 45, 63 (2012).

[14] B. Corominas-Murtra and R. V. Solé, Phys. Rev. E 82, 011102 (2010).

[15] X. Gabaix, Quart. J. Econom. 114, 739 (1999).

[16] A. Hernando, C. Vesperinas, and A. Plastino, Physica A 389, 490 (2010).

[17] A. Hernando et al., Eur. Phys. J. B 76, 87 (2010).

[18] E. W. Weisstein, "Benford's Law." From MathWorldA Wolfram Web Resource (2012), mathworld.wolfram.com/ BenfordsLaw.html.

[19] F. Benford, Proc. Amer. Philos. Soc. 78, 551 (1938).

[20] [http://en.wikipedia.org/wiki/Log-normal] (2012).

[21] J. S. Huxley, Problems of Relative Growth (Methuen, London, 1932).

[22] F. Black and M. Scholes, J. Polit. Economy 81, 637 (1973); S. M. Ross, Introduction to Probability Models, 9th ed. (Academic Press, New York, 2007).
[23] H. Rozenfeld et al., Proc. Nat. Acad. Sci. 105, 18702 (2008).

[24] S. Ree, Phys. Rev. E 73, 026115 (2006).

[25] W. J. Reed and B. D. Hughes, Phys. Rev. E 66, 067103 (2002).

[26] S. Galam, J. Stat. Phys. 61, 943 (1990).

[27] http://www.urbansim.org.

[28] M. Batty, Cities and Complexity (MIT Press, Cambridge, 2005).

[29] C. Castellano, S. Fortunato, and V. Loreto, Rev. Mod. Phys. 81, 591 (2009).

[30] S. Fortunato and C. Castellano, Phys. Rev. Lett. 99, 138701 (2007).

[31] S. Galam, Physica A 285, 66 (2000).

[32] A. Hernando and A. Plastino, Eur. Phys. J. B 85, 293 (2012).

[33] A. Hernando, R. Hernando, A. Plastino, and A. R. Plastino, J. R. Soc. Interface 10, 20120758 (2013).

[34] A. Hernando, A. Plastino, and A. R. Plastino, Eur. Phys. J. B 85, 147 (2012).

[35] A. Hernando and A. Plastino, Phys. Lett. A (2012), doi: 10.1016/j.physleta.2012.10.054.

[36] National Statistics Institute of Spain INE Government of Spain (2012), www.ine.es.

[37] A. Katz, Principles of Statistical Mechanics (Freeman, San Francisco, 1967).

[38] C. Tsallis, Introduction to Nonextensive Statistical Mechanics (Springer, New York, 2009).

[39] Census Bureau, Government of USA (2012), www.census.gov.

[40] R. I. M. Dunbar, J. Hum. Evo. 20, 469 (1992); Beh. Brain Sci. 16, 681 (1993).

[41] Vâl d'Aoûta, Wikivoyage (2012), de.wikivoyage.org/wiki/ Datei:V\%C3\%A21_d\%27Ao\%C3\%BBta.png.

[42] Marshall Islands location map, Wikimedia Commons (2012), commons.wikimedia.org/wiki/File:Marshall_Islands_ location_map.svg.

[43] Economic Policy, Planning and Statistics Office, Republic of the Marshall Islands (2012), http://www.spc.int/prism/ country/mh/stats/.

[44] National Statistical Institute ISTAT, Government of Italy (2012), en.istat.it.

[45] A. Hernando and A. Plastino, arXiv:1207.6504 (2012). 